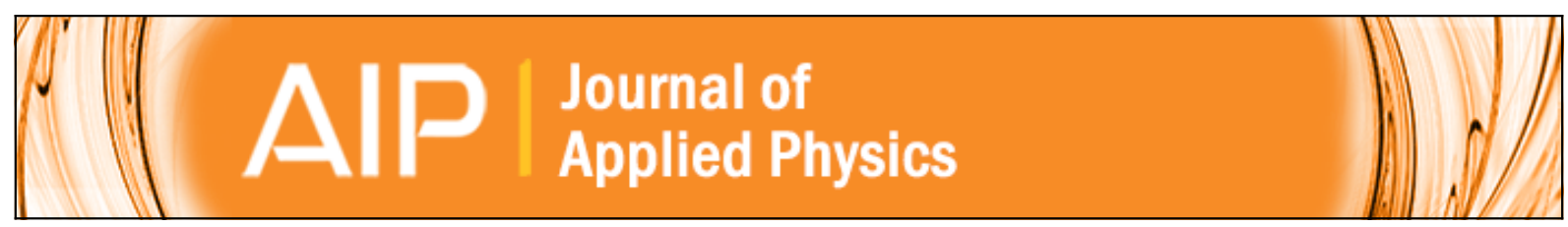

\title{
Vacancies and defect levels in III-V semiconductors
}

H. A. Tahini, A. Chroneos, S. T. Murphy, U. Schwingenschlögl, and R. W. Grimes

Citation: Journal of Applied Physics 114, 063517 (2013); doi: 10.1063/1.4818484

View online: http://dx.doi.org/10.1063/1.4818484

View Table of Contents: http://scitation.aip.org/content/aip/journal/jap/114/6?ver=pdfcov

Published by the AIP Publishing

$\stackrel{A}{A} \mathbb{P} P$ Re-register for Table of Content Alerts

Create a profile.

Sign up today! 


\title{
Vacancies and defect levels in III-V semiconductors
}

\author{
H. A. Tahini, ${ }^{1, a)}$ A. Chroneos, ${ }^{2, b)}$ S. T. Murphy, ${ }^{1}$ U. Schwingenschlögl, ${ }^{3}$ and R. W. Grimes ${ }^{1, c)}$ \\ ${ }^{1}$ Department of Materials, Imperial College London, London SW7 2AZ, United Kingdom \\ ${ }^{2}$ Materials Engineering, The Open University, Milton Keynes MK7 6AA, United Kingdom \\ ${ }^{3}$ PSE Division, KAUST, Thuwal 23955-6900, Kingdom of Saudi Arabia
}

(Received 21 May 2013; accepted 31 July 2013; published online 13 August 2013)

\begin{abstract}
Using electronic structure calculations, we systematically investigate the formation of vacancies in III-V semiconductors ( $\mathrm{III}=\mathrm{Al}, \mathrm{Ga}$, and $\mathrm{In}$ and $\mathrm{V}=\mathrm{P}, \mathrm{As}$, and $\mathrm{Sb}$ ), for a range of charges $(-3 \leq q \leq 3)$ as a function of the Fermi level and under different growth conditions. The formation energies were corrected using the scheme due to Freysoldt et al. [Phys. Rev. Lett. 102, 016402 (2009)] to account for finite size effects. Vacancy formation energies were found to decrease as the size of the group $\mathrm{V}$ atom increased. This trend was maintained for Al-V, Ga-V, and In-V compounds. The negative- $U$ effect was only observed for the arsenic vacancy in GaAs, which makes a charge state transition from +1 to -1 . It is also found that even under group III rich conditions, group III vacancies dominate in AlSb and GaSb. For InSb, group V vacancies are favoured even under group V rich conditions. (C) 2013 AIP Publishing LLC.

[http://dx.doi.org/10.1063/1.4818484]
\end{abstract}

\section{INTRODUCTION}

The III-V family of semiconductors has been researched intensively for the past three decades. In particular, gallium arsenide (GaAs) is the most studied semiconductor after silicon ${ }^{1}$ and many of its bulk properties are well understood and characterised. $^{2}$ The interest in these materials is due to their wide range of applications. For instance, gallium antimonide (GaSb) is of interest for mid-infrared optoelectronics and could play an important role in nanoelectronic devices. ${ }^{3} \mathrm{GaAs}$, indium arsenide (InAs), and their ternary alloys are increasingly used in fabricating high speed electronics and they are at the heart of the International Technology Roadmap for Semiconductors. ${ }^{4,5}$ Direct band gap materials such as InAs, GaSb, and indium phosphide (InP), with band gaps of $0.42,0.81$, and $1.42 \mathrm{eV}$, respectively, ${ }^{6}$ make them efficient light emitters, particularly in lasers and light emitting diodes. Indirect band gap compounds (for example, aluminium arsenide (AlAs) and aluminium antimonide $(\mathrm{AlSb})$ ) find use in radiation detectors, where the indirect band gap suppresses radiative recombination, allowing the electron-hole pair that was generated by an incoming photon more time to be detected.

With the constant downscaling and miniaturisation of electronic devices, it is always crucial to understand the nature and the evolution of the defects formed during the growth processes and the interaction of these defects with various doping species. The most simple case, that of selfdiffusion, is still not fully understood.

Atomic scale simulations are used extensively in studying III-V compounds. ${ }^{7,8,20}$ Nevertheless, there are still many open questions related to the formation and migration of intrinsic and extrinsic defects and the ionization levels of the various species.

\footnotetext{
a)h.tahini@imperial.ac.uk

b) alex.chroneos@open.ac.uk

c)r.grimes@imperial.ac.uk
}

The principle aim of this paper is to provide a consistent and systematic survey of vacancies in binary III-V compounds. The paper is organised as follows: in Sec. II A, we discuss the methodology in terms of the computational parameters employed. Section II B briefly discusses the various charge correction schemes and our method of choice. Results regarding each III-V semiconductor are presented in Sec. III. Finally, we make some remarks concerning trends, in terms of electronegativity and covalent atom radii, and draw conclusions.

\section{METHODOLOGY}

\section{A. Computational details}

The Vienna $A b$ initio Simulation Package ${ }^{9}$ was employed to predict defect formation energies, atomic and electronic structures. The generalised gradient approximation (GGA) with electron exchange and correlation is described according to the Perdew, Burke, and Ernzerhof (PBE) formalism. ${ }^{10}$ Pseudopotentials were generated according to the projector augmented-wave (PAW) method ${ }^{11}$ and a plane-wave basis with a cut-off energy of $400 \mathrm{eV}$ was used. 3 and 5 electrons were treated as valence for group III and $\mathrm{V}$, respectively. We performed tests on $\mathrm{GaAs}, \mathrm{GaSb}$, and InSb using 13 and 15 electrons group III and $\mathrm{V}$, respectively, and found that vacancy formation energies change by no more than $0.05-0.1 \mathrm{eV}$ confirming the ability of the PAW method to account better for core electrons. A few calculations were carried out using 64 atom supercells but the majority employed 216 atom supercells. The Brillouin zone was sampled according to the Monkhorst-Pack scheme ${ }^{12}$ using meshes of $3 \times 3 \times 3$ and $2 \times 2 \times 2$ for the 64 and 216 supercells, respectively, in order to maintain a k-point density as constant as possible across the various supercells. Energies and forces were iterated until convergence of $1 \times 10^{-5} \mathrm{eV}$ and $1 \times 10^{-3} \mathrm{eV} / \AA$ were achieved, respectively. The calculations were all spin-polarised and the simulations of the defect 
containing supercells were carried out under constant volume conditions (i.e., lattice parameters and angles were fixed) while allowing atoms to fully relax. Formation energies $\left(E^{f}\right)$ were calculated based on the formulation of Zhang and Northrup ${ }^{13}$ as detailed by El-Mellouhi and Mousseau ${ }^{14}$

$$
\begin{aligned}
E^{f}= & E_{\text {tot }}(D, q)-E_{\text {tot }}(\text { perfect })+\sum_{\alpha} n_{\alpha} \mu_{\alpha}+q \mu_{e} \\
& \pm 1 / 2 \Delta \mu+E_{\text {corr }},
\end{aligned}
$$

where $E_{t o t}(D, q)$ is the energy of the defective cell with a charge $q$ and $E_{\text {tot }}$ (perfect) is the energy of the perfect cell. $n_{\alpha}$ are the numbers of atoms added/removed multiplied by their corresponding chemical potentials, $\mu_{\alpha} . \mu_{e}$ is the Fermi level referenced to the top of the valence band. $\Delta \mu$ is the chemical potential difference where the upper sign stands for group $\mathrm{V}$ vacancies and the lower sign stands for group III vacancies. This term has upper and lower bounds given by $-\Delta H \leq \Delta \mu$ $\leq+\Delta H$, where $\Delta H$ represents the heat of formation of a compound and is given by

$$
\Delta H_{\mathrm{III}-\mathrm{V}}=\mu_{\mathrm{III}-\mathrm{V}}^{\mathrm{bulk}}-\mu_{\mathrm{III}}^{\mathrm{bulk}}-\mu_{\mathrm{V}}^{\mathrm{bulk}} .
$$

Finally, $E_{\text {corr }}$ is a formation energy correction term.

Spin-orbit interactions were investigated in 64 atom supercells. The effect this had on the formation energies was on the order of few $\mathrm{meV}$ in agreement with previous work on a variety of semiconductors and insulators. ${ }^{15-18}$ As such, spin-orbit interactions were omitted in subsequent calculations.

In this work, we decided to use PBE rather than hybrid functionals. In several cases, hybrid functionals have been shown to outperform other functionals in describing electronic structure and optical properties of materials, and thus, they were assumed to be accurate and superior in all other cases. This opinion was recently disputed by many authors like Youssef and Yildiz ${ }^{19}$ and Ramprasad et al. ${ }^{20}$ who argued that given the known underestimation of formation energies by PBE, hybrid functionals does overestimate them in many cases. Another reason we chose not to use hybrid functionals is that here our focus is not entirely on the absolute values of the formation energies but rather the trends produced by changes in the composition from group III to group V as will be shown in Sec. III.

\section{B. Finite size corrections}

The effects of using supercells and their image repetitions in 3D are fairly well understood in terms of the consequent spurious interactions. ${ }^{21}$ Nevertheless, the case is complicated by the introduction of charged defects since this results in both elastic and electrostatic interactions between the periodic defective cells. To account for the latter, different schemes were introduced to eliminate these unrealistic interactions. One of the earliest attempts was the MakovPayne correction scheme, ${ }^{22}$ which takes into account the screening introduced by the lattice characterised by the Madelung constant $\left(\alpha_{M}\right)$ and the dielectric constant $(\epsilon)$ on a localized charge $q$, given by

$$
E(L)=E\left(L_{\infty}\right)-\frac{\alpha_{M} q^{2}}{2 \epsilon L}-\frac{2 \pi q Q}{3 \epsilon L^{3}}
$$

where $Q$ is the quadrupole moment of the defect charge and $L$ is the defect-defect separation. Also, the introduction of a defect calls for an alignment, $q \Delta V$, between the electrostatic potentials of the defective and perfect (reference) cells. Recently, Freysoldt et al. ${ }^{23,24}$ described a more rigorous and practical approach to this problem. It involves calculating the interaction energies between the periodic repetitions and also the interaction energy of the compensating background with the defect potential, to give a screened lattice energy, $E_{q}^{\text {latt }}$. The defect potential can be deconvoluted into a longrange and a short-range potential, for which the latter decays to zero far away from the defect (see Ref. 23), leading into a correction term

$$
E_{\text {corr }}=E_{q}^{\text {latt }}-q \Delta V_{q / 0}
$$

where $\Delta V_{q / 0}$ is the alignment term between the perfect reference cell and the defective cell. The connection between this scheme and the Makov-Payne method ${ }^{22}$ was established by Komsa et $a .^{21}$ In this study, the scheme due to Freysoldt et al. ${ }^{23}$ was adopted to correct for charged defect interactions due to its practicality as it only involves knowing the electrostatic potentials for the perfect and defective cells, which are obtained in a fully $a b$ initio manner without reliance on external parameters and without the need for carrying out several supercell calculations as is necessary with other methods. $^{25}$

\section{RESULTS}

\section{A. Lattice, elastic, thermodynamic, and electronic properties}

The effectiveness of the computational approach to predicting property trends is first tested by calculating lattice parameters, thermodynamic, electronic, and elastic properties of III-V binary compounds (see Table I). Lattice parameters are all in agreement with experimental data and, as expected from GGA calculations, are all slightly overestimated in comparison with experiments. The calculated heats of formation as defined by Eq. (2) are compared with experimental values and are all within the level of accuracy expected using this technique. ${ }^{26,27}$ Conversely, predicted dielectric constants are both larger and smaller than experimental values. Compounds incorporating larger atoms have higher dielectric constants. The elastic constants $\left(c_{11}, c_{12}\right.$, and $\left.c_{44}\right)$ are shown in Table I. Again the predictions follow the experimental data with compounds (AlP, AlSb, GaP, and GaSb) showing very good agreement. Overall, the computational approach is seen to reproduce a range of perfect lattice properties including those (i.e., elastic and dielectric constants) that are important indicators of the ability to model the response of a lattice to the incorporation of a defect.

\section{B. Charge correction scheme}

As mentioned above, in order to correct for the spurious interactions between the periodic charged defects, we 
TABLE I. The calculated lattice parameters $(\AA)$, heats of formation, $\Delta H$ (eV/formula unit), bandgaps $(\mathrm{eV})$, dielectric constants, elastic constants $\left(c_{11}, c_{12}\right.$, and $\left.c_{44}(\mathrm{GPa})\right)$, and bulk moduli $(\mathrm{GPa})$ with their equivalent experimental values. ${ }^{28,29}$

\begin{tabular}{|c|c|c|c|c|c|c|c|c|c|c|c|c|c|c|c|c|}
\hline & \multicolumn{2}{|c|}{ Lattice parameter } & \multicolumn{2}{|c|}{ Heat of formation } & \multicolumn{2}{|c|}{ Band gap } & \multicolumn{2}{|c|}{ Dielectric constant } & \multicolumn{2}{|c|}{$c_{11}$} & \multicolumn{2}{|c|}{$c_{12}$} & \multicolumn{2}{|c|}{$c_{44}$} & \multicolumn{2}{|c|}{ Bulk modulus } \\
\hline & $\begin{array}{l}\text { This } \\
\text { work }\end{array}$ & $\begin{array}{c}\text { Exp } \\
(\sim 300 \mathrm{~K})\end{array}$ & $\begin{array}{l}\text { This } \\
\text { work }\end{array}$ & $\begin{array}{c}\text { Exp } \\
(\sim 300 \mathrm{~K})\end{array}$ & $\begin{array}{l}\text { This } \\
\text { work }\end{array}$ & $\begin{array}{l}\operatorname{Exp} \\
(\sim 0 \mathrm{~K})\end{array}$ & $\begin{array}{l}\text { This } \\
\text { work }\end{array}$ & $\begin{array}{l}\text { Exp } \\
(\sim 0 \mathrm{~K})\end{array}$ & $\begin{array}{l}\text { This } \\
\text { work }\end{array}$ & Exp & $\begin{array}{l}\text { This } \\
\text { work }\end{array}$ & Exp & $\begin{array}{l}\text { This } \\
\text { work }\end{array}$ & Exp & $\begin{array}{l}\text { This } \\
\text { work }\end{array}$ & Exp \\
\hline AlP & 5.51 & 5.46 & -1.32 & -1.73 & 1.63 & 2.51 & 7.69 & 9.80 & 132.35 & 132.00 & 67.88 & 63.00 & 61.75 & 61.50 & 89.37 & 86.00 \\
\hline AlAs & 5.73 & 5.66 & -0.98 & -1.25 & 1.50 & 2.23 & 9.08 & $\ldots$ & 112.75 & 125.00 & 58.71 & 53.40 & 52.60 & 54.20 & 76.72 & 77.26 \\
\hline $\mathrm{AlSb}$ & 6.23 & 6.14 & -0.33 & -0.52 & 1.23 & 1.69 & 12.68 & 11.21 & 84.91 & 89.39 & 45.03 & 44.27 & 37.66 & 41.55 & 58.32 & 59.31 \\
\hline $\mathrm{GaP}$ & 5.53 & 5.45 & -0.86 & -0.91 & 1.51 & 2.35 & 9.70 & 10.75 & 139.61 & 141.20 & 69.42 & 62.53 & 66.07 & 70.47 & 92.82 & 88.75 \\
\hline GaAs & 5.76 & 5.65 & -0.70 & -0.74 & 0.05 & 1.52 & 14.02 & 12.90 & 115.33 & 118.77 & 57.91 & 53.72 & 54.76 & 59.44 & 77.05 & 75.40 \\
\hline $\mathrm{GaSb}$ & 6.22 & 6.10 & -0.32 & -0.43 & 0.20 & 0.81 & 16.95 & 15.70 & 86.12 & 88.39 & 43.15 & 40.33 & 39.93 & 43.16 & 57.47 & 56.35 \\
\hline $\mathrm{InP}$ & 6.00 & 5.86 & -0.48 & -0.92 & 0.41 & 1.42 & 10.82 & 12.61 & 103.14 & 102.20 & 67.29 & 57.60 & 40.03 & 46.00 & 79.24 & 72.46 \\
\hline InAs & 6.21 & 6.05 & -0.49 & -0.61 & $\ldots$ & 0.42 & 15.75 & 15.15 & 86.92 & 83.29 & 56.44 & 45.26 & 34.20 & 39.59 & 66.60 & 57.94 \\
\hline InSb & 6.65 & 6.47 & -0.26 & -0.32 & $\ldots$ & 0.24 & 18.74 & 17.88 & 67.27 & 67.20 & 42.02 & 36.70 & 26.86 & 30.20 & 50.44 & 46.86 \\
\hline
\end{tabular}

employ the correction scheme due to Freysoldt et al. ${ }^{23,24}$ The technique has been demonstrated to efficiently correct for charged defect interactions in smaller supercells. ${ }^{30,31}$ Here, we performed tests on charged $\mathrm{Ga}$ and $\mathrm{P}$ vacancies in GaP using 64 and 216 atom supercells (see Fig. 1). The uncorrected energies derived from the two cells clearly diverge for higher charges. The application of the charge correction scheme brings these values into agreement, within $\sim 0.1 \mathrm{eV}$ per vacancy.

In the following subsections, we will present the corrected results along with their interpretation focusing on the stoichiometric conditions of the crystal. The figures also show that the formation energies change under different growth conditions although we will initially discuss defects under stoichiometric conditions.
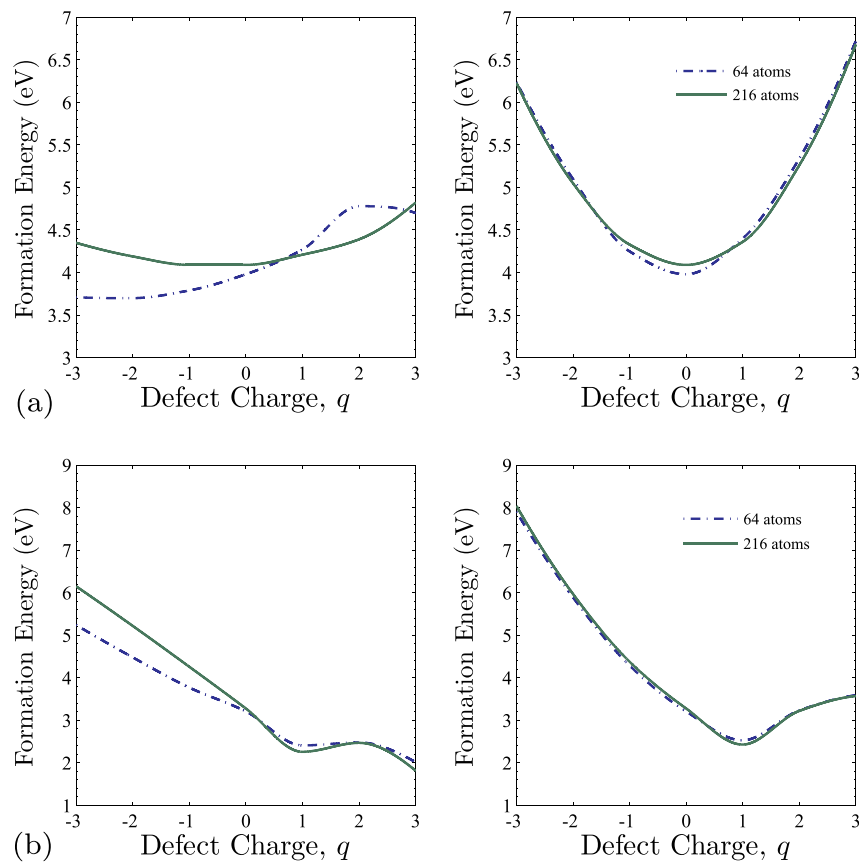

FIG. 1. Formation energies of (a) $\mathrm{Ga}$ and (b) $\mathrm{P}$ vacancies in $\mathrm{GaP}$ using 64 atom and 216 atom supercells. The left panels are the uncorrected energies while those on the right are the formation energies corrected using the correction scheme due to Freysoldt et al. ${ }^{23,24}$

\section{Aluminum-V compounds}

\section{Aluminium phosphide}

AlP is an indirect band gap semiconductor $\left(\mathrm{E}_{\mathrm{g}}=2.5 \mathrm{eV}\right)$ that has found application in light emitting diodes. Unlike other III-V materials, this compound has not been widely studied and as such many defect properties are incompletely understood. A few studies ${ }^{32,33}$ were carried out on AlP that mostly focused on the electronic structure. Fig. 2(a) shows the formation energies of vacancies in AlP for the charge that is most likely to form (i.e., of lowest energy at a given value of the Fermi level). Thus, aluminium vacancies are most stable in their neutral, $-1,-2$, or -3 charge states depending on the level of doping in the material. Positive charge states have higher formation energies and are thus not likely to form. The formation energy of $V_{\mathrm{Al}}^{0}$ is $4.42 \mathrm{eV}$ under stoichiometric conditions. This defect begins to decrease in concentration as the charged defect $V_{\mathrm{Al}}^{q}$ starts to form as the Fermi level increases. The formation energies of charged defects can fall as low as $1.26 \mathrm{eV}$ (for $V_{\mathrm{Al}}^{-3}$ ). The defect energy transition levels, $\epsilon(0 /-)$ and $\epsilon(-/=)$, occur at $0.81 \mathrm{eV}$ and $1.42 \mathrm{eV}$ above the valence band and $\epsilon(=/ \equiv)$ occurs at $0.38 \mathrm{eV}$ below the conduction band. For all charges, $V_{\mathrm{Al}}^{q}$ exhibits $T_{d}$ point group symmetry.

Phosphorous vacancies occur in the $+1,0,-1,-2$ charge states. Under extreme p-doping conditions, $V_{\mathrm{P}}^{+1}$ will have a formation energy of $\sim 2.61 \mathrm{eV}$; this will keep rising with increasing $\mu_{e}$ until the neutral vacancy becomes dominant under nearly intrinsic doping conditions, with a formation energy of $3.88 \mathrm{eV}$. The lower formation energy of $V_{\mathrm{P}}^{+1}$ implies that up to $\mu_{e}=1.2 \mathrm{eV}, \mathrm{P}$ vacancies will dominate in AlP, and beyond this $V_{\mathrm{Al}}^{-2}$ and $V_{\mathrm{Al}}^{-3}$ are more easily formed.

\section{Aluminium arsenide}

AlAs, with a $2.23 \mathrm{eV}$ indirect band gap, is important for high electron mobility transistors and optoelectronic devices. ${ }^{6}$ It exhibits trends similar to those of AlP in terms of what charge states are favourable and the dominant vacancy at a given doping level. $V_{\mathrm{Al}}^{0}$ is most stable under heavy to moderate p-doping with a formation energy of $3.62 \mathrm{eV}$. 

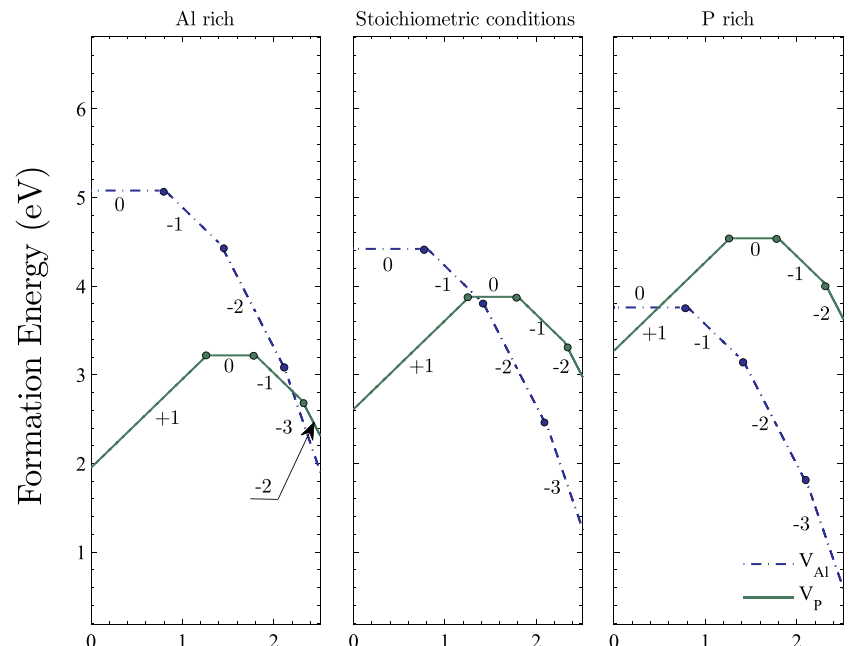

(a) Fermi Level, $\mu_{e}(\mathrm{eV})$
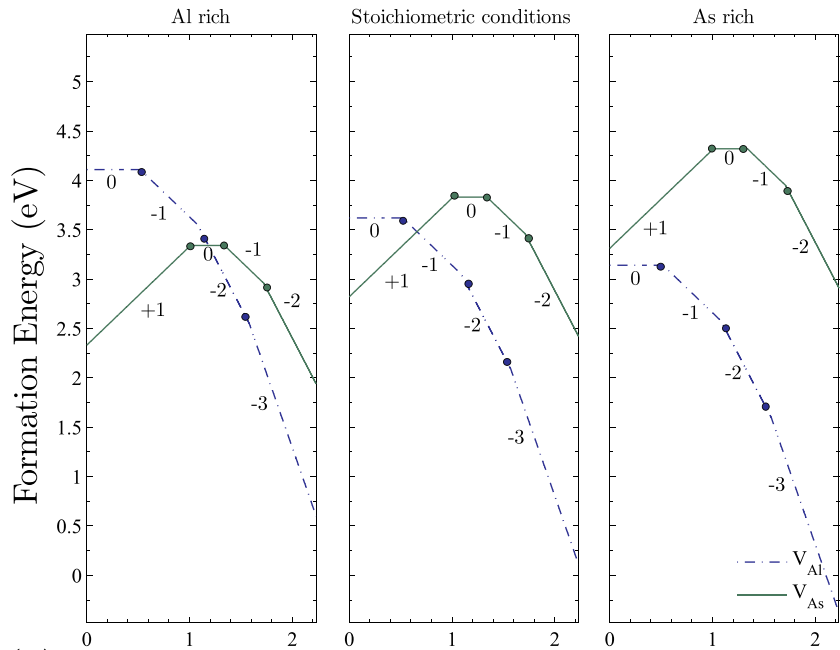

(b)

Fermi Level, $\mu_{e}(\mathrm{eV})$
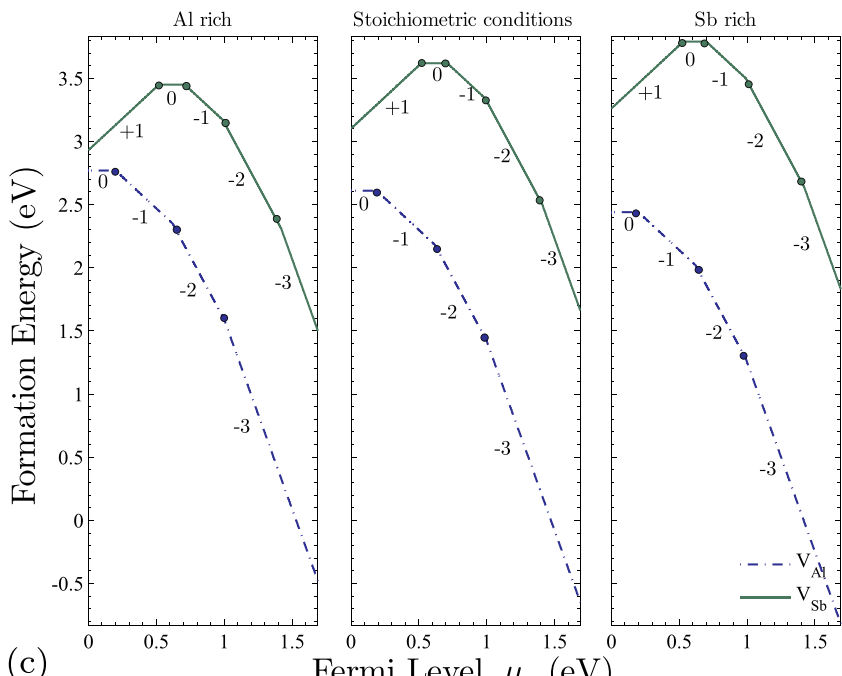

Fermi Level, $\mu_{e}(\mathrm{eV})$

FIG. 2. Lowest energy vacancy formation energies for Al-V compounds assuming the most stable charge state (neutral or charged) as a function of the Fermi level.

Under intrinsic conditions, $V_{\mathrm{Al}}^{-1}$ and $V_{\mathrm{Al}}^{-2}$ are most likely to compete as they both have similar formation energies but, as would be expected, at higher Fermi level, the more negative charge state will become more prevalent. The $V_{\mathrm{Al}}^{q}$ defect level transitions occur at or near the middle of the band gap implying that they are all deep level traps. The point group symmetry of $V_{\mathrm{Al}}^{q}$ in all the charge states considered here is $T_{d}$.

$V_{\mathrm{As}}^{q}$ favours the +1 charge state under p-doping up to intrinsic levels where $V_{\mathrm{As}}^{0}$ with a formation energy of $3.83 \mathrm{eV}$ prevails. With higher n-doping levels, the vacancy captures more electrons moving from $0,-1$ to -2 with formation energies reaching $2.42 \mathrm{eV}$ in highly n-doped regimes. This is also accompanied by a series of changes in point group symmetry from $D_{2 d}$ for $V_{\mathrm{As}}^{0}$ to $C_{2 v}$ for $V_{\mathrm{As}}^{-1}$ and back to $D_{2 d}$ for $V_{\mathrm{As}}^{-2}$. Their formation energies remain, however, higher than the corresponding values for $V_{\mathrm{Al}}^{q}$, which for most of the Fermi level region maintain a difference of about $1 \mathrm{eV}$ from $V_{\mathrm{As}}^{q}$.

\section{Aluminium antimonide}

Similar to AlP and AlAs, AlSb has an indirect band gap of $1.69 \mathrm{eV}$ with applications in long-wavelength optoelectronic and photon detectors. ${ }^{34}$ Fig. 2(c) suggests that $V_{\mathrm{Al}}^{0}$ appears only briefly for a Fermi level close to the valence band. This then gives way to $-1,-2$, and the -3 charge states. $V_{\mathrm{Al}}^{0}$ has a formation energy of $2.61 \mathrm{eV}$, which is the same value obtained by Åberg et al. ${ }^{35}$ The formation energies for negatively charged states fall until, under heavy doping conditions, $V_{\mathrm{Al}}^{-3}$ will attain a negative energy of about $-0.5 \mathrm{eV}$ similar to values predicted by $\mathrm{Du}^{36}$ This implies that under heavy $\mathrm{n}$-doping conditions, it will be energetically favourable for $V_{\mathrm{Al}}^{-3}$ to form.

$V_{\mathrm{Sb}}^{q}$ has higher formation energies than $V_{\mathrm{Al}}^{q}$ across the entire bandgap. Under $\mathrm{p}$-doping, the +1 charge state will form and remains stable up to $\mu_{e}=0.55 \mathrm{eV}$ where the neutral vacancy supersedes it with a formation energy of $3.62 \mathrm{eV}$. This value is only slightly different to that reported by Åberg et al. ${ }^{35}$ who calculated an equivalent energy of $3.42 \mathrm{eV}$. 


\section{Gallium-V compounds}

\section{Gallium phosphide}

$\mathrm{GaP}$ has a $2.35 \mathrm{eV}$ indirect band gap, which when doped with nitrogen could be used in light emitting diodes. The vacancies and defect levels have been studied from both experimental and computational points of view. ${ }^{37-40} V_{\mathrm{Ga}}^{0}$ has a formation energy of $4.09 \mathrm{eV}$, which is in good agreement with the value of $4.17 \mathrm{eV}$ reported by Höglund et al. ${ }^{40}$ The occurrence of $V_{\mathrm{Ga}}^{0}$ is only brief with respect to $\mu_{e}$, which then favours more negative charged states. In the first half of the band gap, $V_{\mathrm{Ga}}^{-1}$ and $V_{\mathrm{Ga}}^{-2}$ will be more likely to form, which, under close to intrinsic doping conditions, gives way to $V_{\mathrm{Ga}}^{-3}$ which dominates up to extreme n-doping conditions. The stable transition levels $\epsilon(0 /-), \epsilon(-/=)$ and $\epsilon(=/ \equiv)$ occur at $0.24,0.72$, and $1.18 \mathrm{eV}$, respectively, above the valence band and hence form shallow and deep defect transition levels.

$V_{\mathrm{P}}^{+1}$ starts from the top of the valence band with a formation energy of $2.43 \mathrm{eV}$ and continues to about $0.9 \mathrm{eV}$ above the valence band when it captures an electron forming
$V_{\mathrm{P}}^{0}$ with an energy of $3.28 \mathrm{eV}$, in good agreement with the value predicted by Höglund et al. ${ }^{40}$ of $3.33 \mathrm{eV}$. These species appears to be stable under light p-doping beyond which -1 , -2 , and -3 charge states form, respectively. The defect transition levels $\epsilon(+/ 0), \epsilon(0 /-), \epsilon(-/=)$ occur at $0.85,1.10$, and $1.59 \mathrm{eV}$ above the valence band, respectively, and $\epsilon(=/ \equiv)$ at $0.28 \mathrm{eV}$ below the conduction band.

Both vacancies $\left(V_{\mathrm{Ga}}^{q}\right.$ and $\left.V_{\mathrm{P}}^{q}\right)$ maintain perfect $T_{d}$ point group symmetry with the exception of $V_{\mathrm{P}}^{0}$ which forms a distorted $C_{1 h}$ structure.

\section{Gallium arsenide}

GaAs has been studied extensively. ${ }^{41-45}$ Its $1.52 \mathrm{eV}$ direct band gap makes it suitable for uses ranging from integrated circuits to solar cells. ${ }^{1}$ Remarkably, Fig. 3(b) indicates an absence of neutral $\mathrm{Ga}$ or As vacancies. $V_{\mathrm{Ga}}^{q}$ does not favour any of the positively charged states and it starts by adopting the -1 charge at the top of the valence band. The defect level transition $\epsilon(-/=)$ occurs at $0.45 \mathrm{eV}$ followed by $\epsilon(=/ \equiv)$ at $0.79 \mathrm{eV}$ above the valence band implying
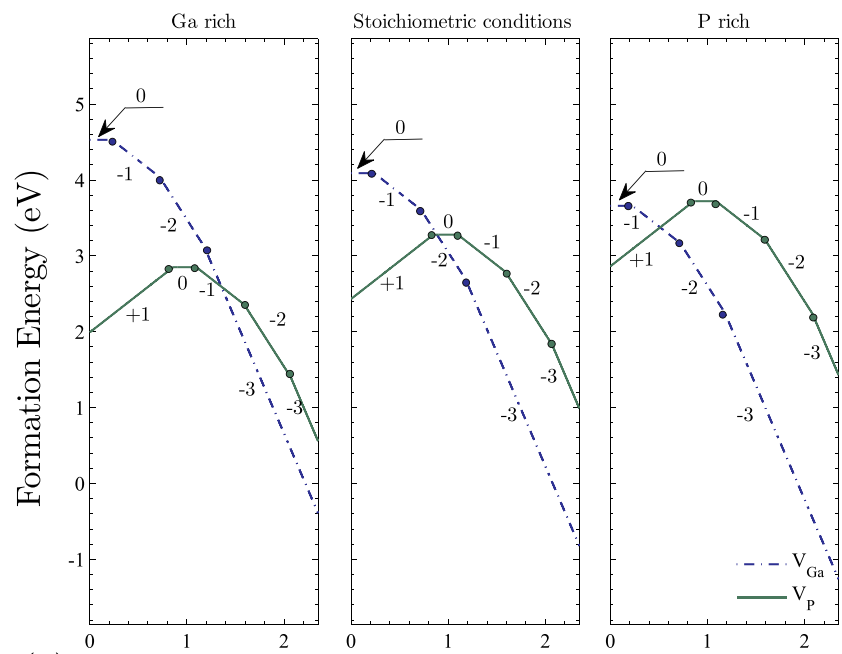

(a)
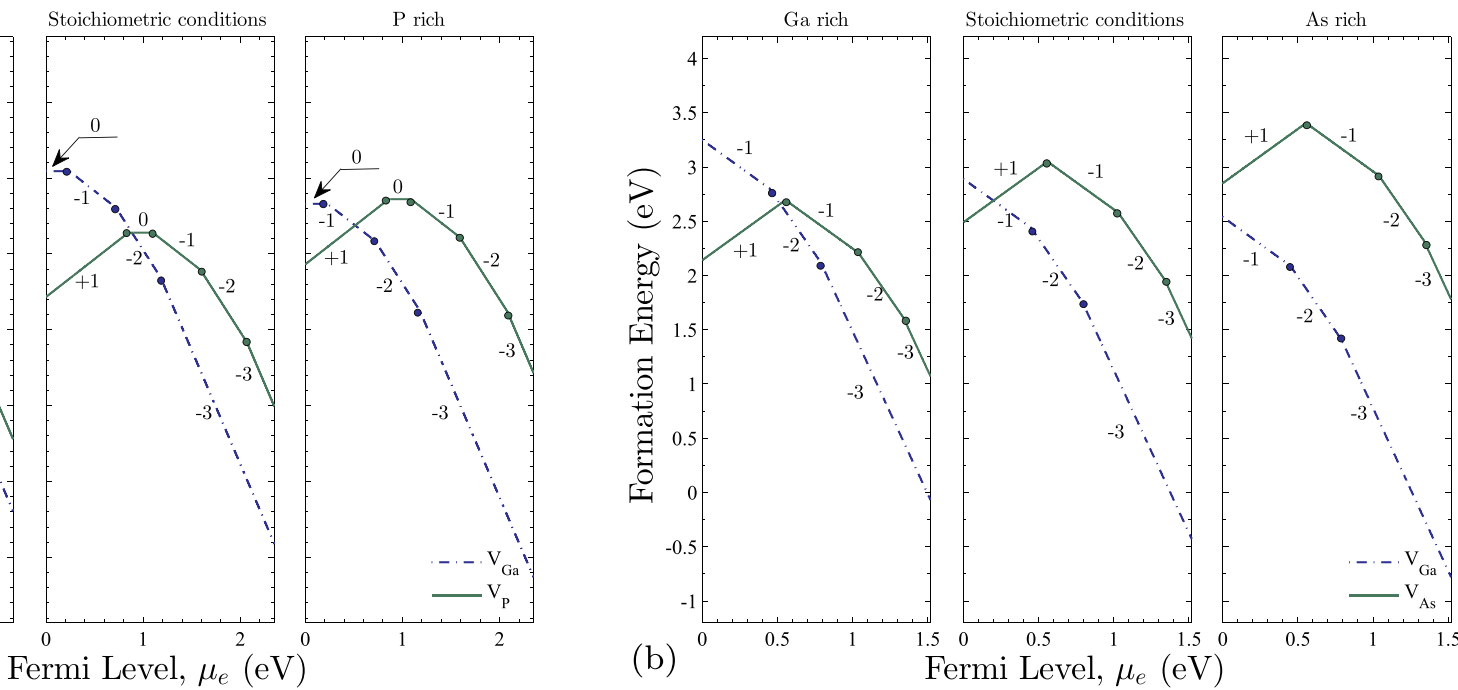

(b)

Fermi Level, $\mu_{e}(\mathrm{eV})$

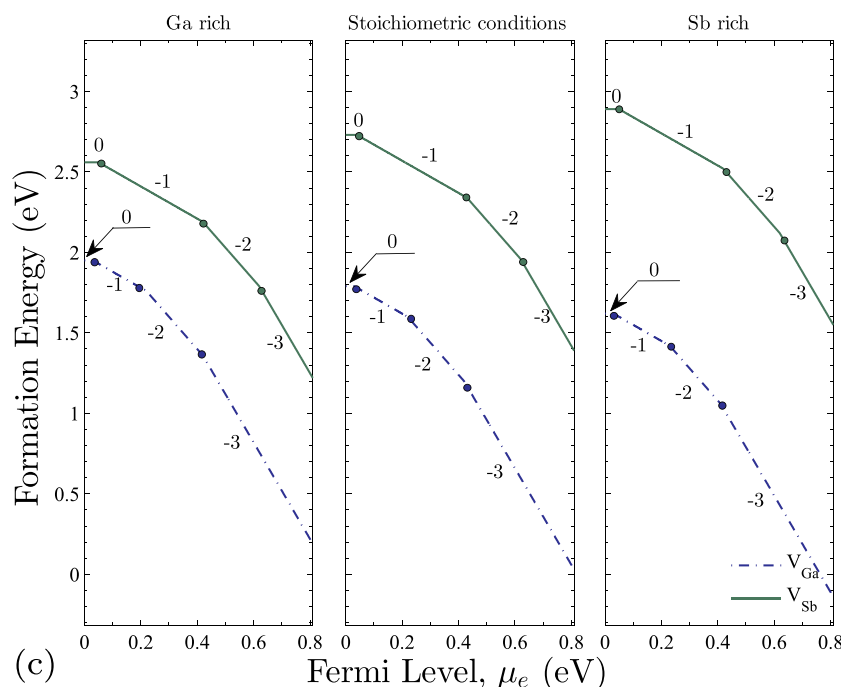

FIG. 3. Lowest energy vacancy formation energies for Ga-V assuming the most stable charge state (neutral or charged) as a function of the Fermi level. 
that under intrinsic and n-doping conditions, $V_{\mathrm{Ga}}^{-3}$ is the most prevalent charge state. $V_{\mathrm{As}}^{+1}$ has the lowest formation energy of $2.49 \mathrm{eV}$ at the top of the valence band, which then captures two electrons forming $V_{\mathrm{As}}^{-1}$. This is known as the negative- $U$ transition, which is consistent with the observations of El-Mellouhi and Mousseau ${ }^{14}$ and in agreement with other works. ${ }^{45-47}$ The negative- $U$ transition, $\epsilon(+/-)$, takes place at $0.56 \mathrm{eV}$ above the valence band. The other two transitions, $\epsilon(-/=)$ and $\epsilon(=/ \equiv)$, occur at 0.49 and $0.18 \mathrm{eV}$ below the conduction band. The neutral and negatively charged As vacancies exhibit $D_{2 d}$ point group symmetry while $V_{\mathrm{As}}^{+1}$ possesses $C_{3 v}$ symmetry. Even though we do not predict its existence, the calculated value for the formation of $V_{\mathrm{As}}^{0}$ is $3.07 \mathrm{eV}$
compared to $2.85 \mathrm{eV}$ predicted by Murphy et al.

\section{Gallium antimonide}

$\mathrm{GaSb}$ is an intermediate band gap material $\left(\mathrm{E}_{g}=0.81 \mathrm{eV}\right)$ that could be used in laser diodes, high frequency devices, and photodetectors with high quantum efficiency. ${ }^{3}$ In GaSb, $V_{\mathrm{Ga}}^{0}$ occurs at doping levels near the top of the valence band with a formation energy of $1.79 \mathrm{eV}$. This undergoes a transition to $V_{\mathrm{Ga}}^{-1}$ at $\epsilon(0 /-)=0.03 \mathrm{eV}$, which renders it a shallow state. With increasing Fermi level, higher negative charge states form leading to two more transitions $\epsilon(-/=)$ and $\epsilon(=/ \equiv)$ at 0.22 and $0.42 \mathrm{eV}$ above the valence band. Under very high n-doping conditions, $V_{\mathrm{Ga}}^{-3}$ will achieve very low formation energies $(\sim 0.05 \mathrm{eV}) . V_{\mathrm{Sb}}^{q}$ follows similar trends to those of $V_{\mathrm{Ga}}^{q} \cdot V_{\mathrm{Sb}}^{0}$ has a formation energy of $2.73 \mathrm{eV}$, which is $0.94 \mathrm{eV}$ higher than $V_{\mathrm{Ga}}^{0}$. The corresponding $\mathrm{Sb}$ vacancies maintain a difference of about 1.2-1.4 eV higher than the Ga vacancies at any given level of $\mu_{e}$ reported here. These large differences in the formation energies between the two species suggest that $V_{\mathrm{Ga}}^{q}$ will dominate and are likely to have much higher concentrations than $V_{\mathrm{Sb}}^{q}$. This has significant consequences for the self-diffusion in GaSb. The prevalence of $V_{\mathrm{Ga}}^{q}$ for all the charge states and values of $\mu_{e}$ considered is consistent with the significantly higher diffusion of $\mathrm{Ga}$ (diffusion mechanism involving $V_{\mathrm{Ga}}$ ) compared with $\mathrm{Sb}^{49,50}$

\section{E. Indium-V compounds}

\section{Indium phosphide}

InP is used as a substrate in optoelectronic devices and as a high-frequency electronic material due to its high electron mobility. ${ }^{51}$ Our calculated value for the formation energy of $V_{\text {In }}^{0}$ is $4.14 \mathrm{eV}$, in good agreement with several previously calculated values. ${ }^{52-54}$ The neutral vacancy is stable above the valence band and in the extreme p-doping regime, which eventually captures an electron forming $V_{\text {In }}^{-1}$ with a transition $\epsilon(0 /-)=0.18 \mathrm{eV}$. The following transitions $\epsilon(-/=)$ and $\epsilon(=/ \equiv)$ occur at 0.61 and $1.08 \mathrm{eV}$, respectively. $V_{\mathrm{P}}^{+1}$ becomes dominant from the top of the valence band with a formation energy of $1.85 \mathrm{eV}$, which is $2.29 \mathrm{eV}$ less than $V_{\mathrm{In}}^{0} \cdot \epsilon(+/ 0)$ occurs at $0.66 \mathrm{eV}$ where $V_{\mathrm{P}}^{0}$ becomes more favourable with a formation energy of $2.51 \mathrm{eV}$. A second transition, $\epsilon(0 /-)$, takes place at $1.03 \mathrm{eV} . V_{\mathrm{P}}^{+1}$ exhibits $T_{d}$ point group symmetry, while $V_{\mathrm{P}}^{0}$ and $V_{\mathrm{P}}^{-1}$ possess $D_{2 d}$ point group symmetry. The lower formation energy of $V_{\mathrm{P}}^{q}$ compared to $V_{\text {In }}^{q}$ for a wide Fermi level range (up to $\mu_{e} \sim 1.23 \mathrm{eV}$ ) implies that until the high $\mathrm{n}$-doping regime $\mathrm{P}$ vacancies will be the dominant species.

\section{Indium arsenide}

InAs has a small direct band gap of $0.42 \mathrm{eV}$ and as such has been used in long-wavelength optoelectronics and electron quantum wells. ${ }^{6}$ The In vacancy forms in three charge states $0,-1$, and -2 . $V_{\text {In }}^{0}$ has a formation energy of $3.01 \mathrm{eV}$ and dominates at the lower end of the Fermi level. At $\epsilon(0 /-)=0.06 \mathrm{eV}, V_{\text {In }}^{-1}$ is favoured and dominates over a wide Fermi level range until $\mu_{e}=0.35 \mathrm{eV}$ whereupon $V_{\mathrm{In}}^{-2}$ forms. However, $V_{\mathrm{In}}^{q}$ remains much higher in energy than $V_{\mathrm{As}}^{q}$, which under p-doping and light n-doping occurs as $V_{\mathrm{As}}^{+1}$ with a formation energy of $2.00 \mathrm{eV}$ at the top of the valence band. $V_{\mathrm{As}}^{+1}$ extends to $\mu_{e}=0.27 \mathrm{eV}$ at which point $V_{\mathrm{As}}^{0}$ forms at a cost of $2.27 \mathrm{eV}$ in agreement with the value $2.30 \mathrm{eV}$ reported by Murphy et $a l^{48}$ The As vacancy maintains a much lower formation energy than $V_{\text {In }}^{q}$ suggesting that this will be the major vacancy defect during the actual growth conditions of the crystal (see Sec. IV).

\section{Indium antimonide}

InSb has one of the smallest band gaps in the III-V family of semiconductors $\left(\mathrm{E}_{\mathrm{g}}=0.24 \mathrm{eV}\right)$ and possesses the highest electron mobility. These properties make it useful in infrared optoelectronics including infrared detectors. ${ }^{55}$ The small band gap limits the possibility of different charge states forming and hence limits the defect level transitions to at most one. For nearly the entire Fermi level range, $V_{\text {In }}^{-1}$ dominates except under extreme doping conditions when the transition $\epsilon(-/=)=0.23 \mathrm{eV}$ results in $V_{\mathrm{In}}^{-2}$ with a formation energy of $2.44 \mathrm{eV}$. On the other hand, $\mathrm{Sb}$ vacancies have much lower formation energies starting with $1.62 \mathrm{eV}$ for $V_{\mathrm{Sb}}^{+1}$ at the top of the valence band. This transforms into $V_{\mathrm{Sb}}^{0}$ at $\mu_{e}=0.03 \mathrm{eV}$ with a formation energy of $1.65 \mathrm{eV}$, which is much lower than those for $V_{\mathrm{In}}^{q}$.

\section{THE INFLUENCE OF GROWTH CONDITIONS: STOICHIOMETRY}

The above analysis relates to the compounds' chemical potential $\Delta \mu=0$, that is, under stoichiometric conditions. Varying $\Delta \mu$ between $-\Delta H$ (group III rich) and $+\Delta H$ (group $\mathrm{V}$ rich) allows investigation of poor and rich growth conditions, which might be present when synthesising the various compounds. In $\mathrm{AlSb}$ and $\mathrm{GaSb}$, the difference between $\mathrm{Al}$ and $\mathrm{Ga}$ vacancies on one side and $\mathrm{Sb}$ vacancies on the other is at least $0.49 \mathrm{eV}$ and $0.94 \mathrm{eV}$, respectively, for stoichiometric conditions. This implies that even under group III rich conditions, $\mathrm{Al}$ and $\mathrm{Ga}$ vacancies remain lower in energy in these two compounds as $E^{f}$ in Eq. (1) will only increase by $+\Delta H / 2$ which is 0.165 and $0.16 \mathrm{eV}$ in $\mathrm{AlSb}$ and $\mathrm{GaSb}$, respectively (see Figs. 2(c) and 3(c)). The current values also indicate an equivalent situation for $\mathrm{InSb}$, where group $\mathrm{V}$ vacancies will still dominate even under group $\mathrm{V}$ rich conditions (see Fig. 4(c)) as $+\Delta H / 2$ in this case is $0.13 \mathrm{eV}$. Thus, for $\mathrm{AlSb}, \mathrm{GaSb}$, and $\mathrm{InSb}$, the dominant vacancy is invariant 

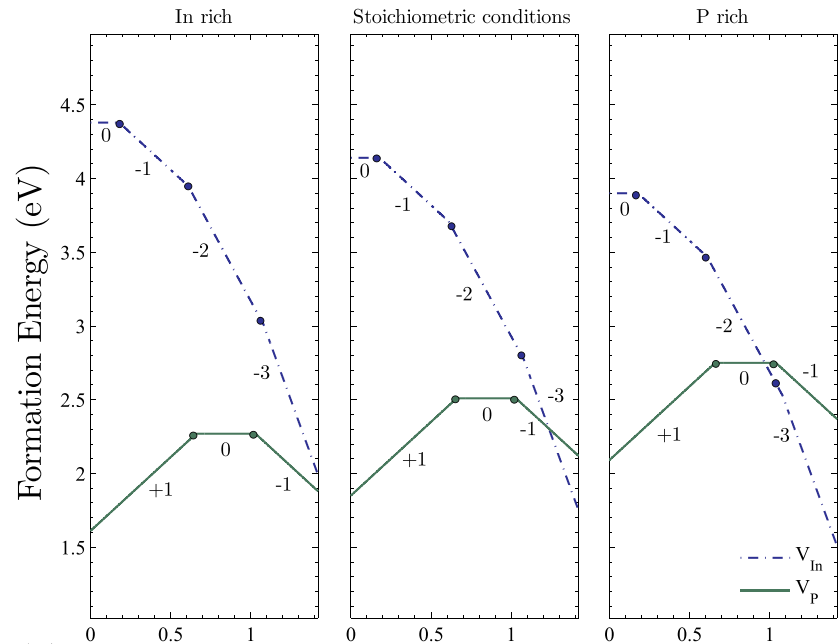

(a)
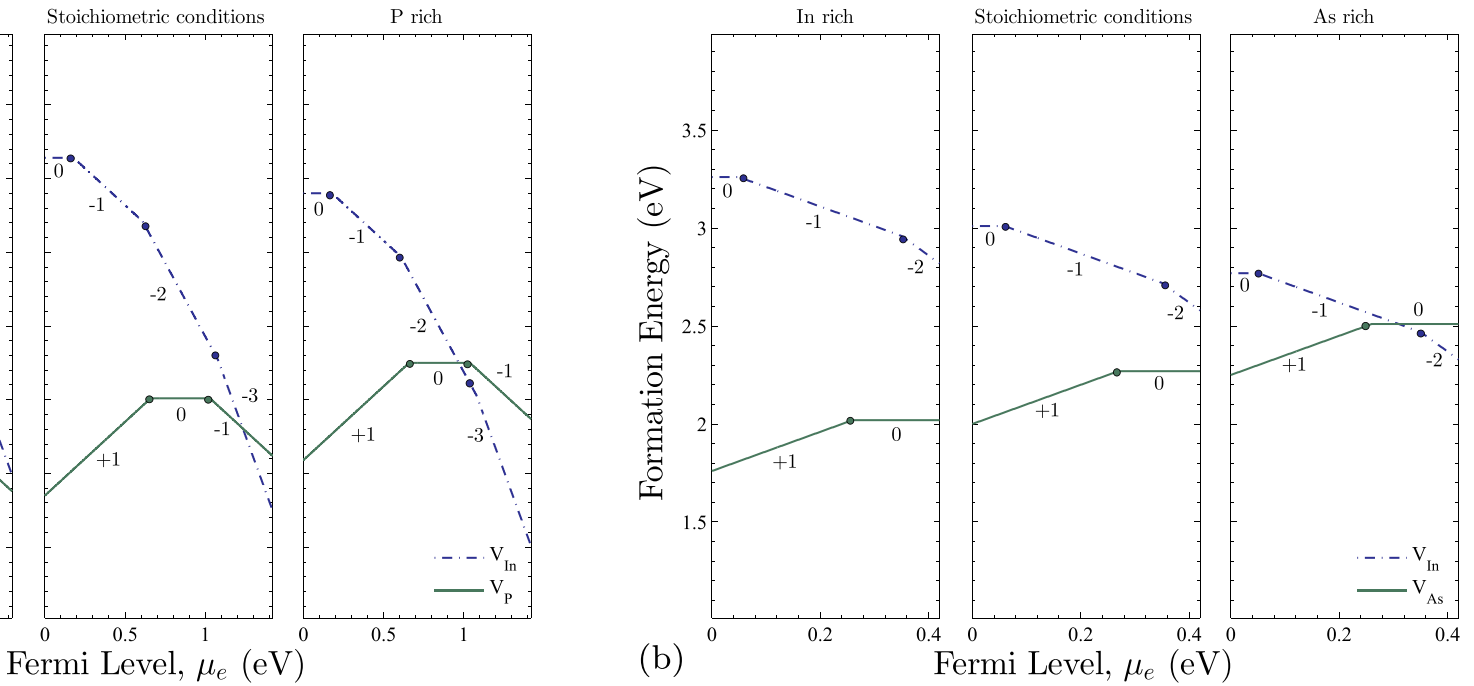

(b)

Fermi Level, $\mu_{e}(\mathrm{eV})$

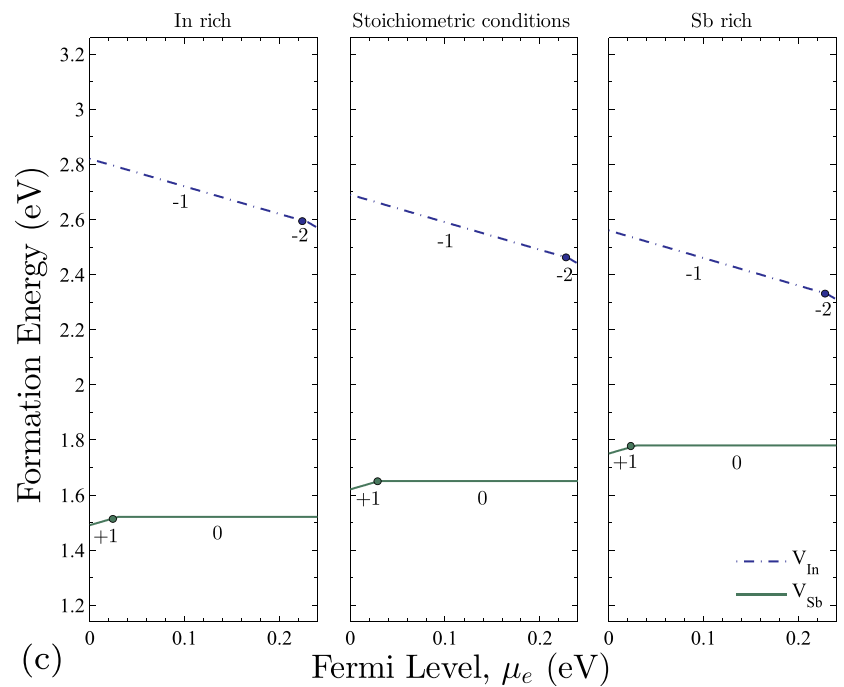

FIG. 4. Lowest energy vacancy formation energies for In-V assuming the most stable charge state (neutral or charged) as a function of the Fermi level.

to the changes in growth conditions across the whole Fermi energy spectrum. For the other six compounds, however, growth conditions can alter the dominant vacancy concentration at specific Fermi energy values though not necessarily for all. For instance, both $V_{\mathrm{Al}}^{q}$ and $V_{\mathrm{As}}^{q}$ dominate in AlAs under $\mathrm{Al}$ rich and stoichiometric conditions depending upon the Fermi level. However, under As rich conditions, $V_{\mathrm{Al}}^{q}$ becomes dominant across the whole band gap (see Fig. 2(b)). GaAs behaves similarly so that while $V_{\mathrm{Ga}}^{q}$ is always dominant under As rich conditions, irrespective of the Fermi level, in $\mathrm{Ga}$ rich conditions, at Fermi levels of less than $0.6 \mathrm{eV}, V_{\mathrm{As}}^{q}$ defects are dominant while above $V_{\mathrm{Ga}}^{q}$ again dominates.

\section{TRENDS IN THE FORMATION ENERGIES}

In order to investigate the influence of the physical properties of group $\mathrm{V}$ atoms on the vacancy formation energies, we categorize the compounds into three sets, Al-V, Ga-V, and $\mathrm{In}-\mathrm{V}$ (where $\mathrm{V}=\mathrm{P}$, As, and $\mathrm{Sb}$ ). The Al-V compounds favour the formation of $V_{\mathrm{Al}}^{q}$ for larger group $\mathrm{V}$ ions. AlP tends to favour $V_{\mathrm{V}}^{q}$ in the first half of the band gap and then favours $V_{\mathrm{Al}}^{q}$ in the second half. Conversely, for AlAs, $V_{\mathrm{Al}}^{q}$ dominates at lower Fermi levels. Finally, in AlSb, $V_{\mathrm{Al}}^{q}$ prevails across the Fermi level. The changes in the formation energies and these trends can be in part attributed to the electronegativities and the covalent bond radii of the constituents. The electronegativities of the group $\mathrm{V}$ elements change as $\mathrm{P}(2.19) \rightarrow \mathrm{As}(2.18) \rightarrow \mathrm{Sb}(2.05)$ and the covalent radii as $\mathrm{P}(1.07 \AA) \rightarrow \mathrm{As}(1.19 \AA) \rightarrow \mathrm{Sb}(1.39 \AA) .{ }^{56}$ A similar trend is seen for $\mathrm{Ga}-\mathrm{V}$ where $V_{\mathrm{Ga}}^{q}$ is the favourable vacancy species and for $\mathrm{GaSb}$, in particular, $V_{\mathrm{Ga}}^{q}$ forms with a much lower energy than $V_{\mathrm{Sb}}^{q}$. However, for In- $\mathrm{V}$, the situation is different: the group $\mathrm{V}$ vacancies are the lower energy species and only in InP does $V_{\text {In }}^{q}$ form and then only under high n-doping conditions.

To further investigate the trends in vacancy formation energies, $V_{\mathrm{III}}^{q}$ and $V_{\mathrm{V}}^{q}$ are shown in Table II for $\mu_{e}=\mathrm{E}_{\mathrm{g}} / 2$, which to a good approximation corresponds to the Fermi level of an intrinsic semiconductor. ${ }^{57}$ The formation energies decrease across the rows of the table, that is, with increasing anion size and decreasing electronegativity. This trend is not surprising given that electrons are less bound to less electronegative atoms (which form weaker bonds that are easier to break, hence forming a vacancy with a relatively lower 
TABLE II. The formation energies of the group III and group V vacancies $(\mathrm{eV})$ for $\mu_{e}=\mathrm{E}_{\mathrm{g}} / 2$ under stoichiometric conditions. The values in parenthesis correspond to the charge of the vacancy under intrinsic conditions.

\begin{tabular}{|c|c|c|c|}
\hline & $\mathrm{P}$ & As & $\mathrm{Sb}$ \\
\hline \multicolumn{4}{|c|}{$V_{\text {III }}^{q}$} \\
\hline $\mathrm{Al}$ & $3.98(-1)$ & $3.00(-2)$ & $1.74(-2)$ \\
\hline $\mathrm{Ga}$ & $2.70(-2)$ & $1.82(-2)$ & $1.23(-2)$ \\
\hline In & $3.51(-2)$ & $2.86(-1)$ & $2.57(-1)$ \\
\hline \multicolumn{4}{|c|}{$V_{\mathrm{V}}^{q}$} \\
\hline $\mathrm{Al}$ & $3.86(+1)$ & $3.83(0)$ & $3.50(-1)$ \\
\hline $\mathrm{Ga}$ & $3.20(-1)$ & $2.85(-1)$ & $2.36(-1)$ \\
\hline In & $2.51(0)$ & $2.21(+1)$ & $1.65(0)$ \\
\hline
\end{tabular}

formation energy (see Table II)). AlP and AlAs anion vacancies have almost the same formation energies of 3.86 and $3.83 \mathrm{eV}$ at intrinsic Fermi levels, which is reflected by the similar anion electronegativities of 2.19 and 2.18 for $\mathrm{P}$ and As, respectively. Conversely, Sb has a much lower electronegativity, which is reflected by the different and lower vacancy formation energy. The same is observed for Ga-V and In-V, where the antimonides always have much lower formation energies than other members in any given set.

Another important feature that can be seen in Figs. 2-4 is the absence of positive charge states for the group III vacancies. Also, group $\mathrm{V}$ vacancies do not exhibit a positive charge beyond +1 . There is some ambiguity in the literature in terms of what are the stable charge states for each of the vacancy defects. For example, our work agrees with $\mathrm{Du}^{36}$ and Åberg et al. ${ }^{35}$ who predict $0,-1,-2$, and -3 charge states for $V_{\mathrm{Al}}^{q}$ in AlSb; however, Åberg et al. ${ }^{35}$ predict charges for $V_{\mathrm{Sb}}^{q}$ ranging from +3 to -2 . In the case of GaAs, our predicted charge states agree with El-Mellouhi and Mousseau ${ }^{14}$ but are at variance with those of Schultz et al. ${ }^{45}$ and Northrup and Zhang ${ }^{58}$ who predict the stability of $V_{\mathrm{As}}^{+3}$. Significantly El-Mellouhi and Mousseau ${ }^{14}$ used the MakovPayne $^{22}$ technique to correct for charged defects, whereas Schultz et al. $^{45}$ and Northrup and Zhang ${ }^{58}$ did not employ such correction schemes. For GaSb, we find that both vacancies will be stable from 0 to -3 charge states depending on the Fermi level, which is in agreement with Virkkala et al. ${ }^{59}$ in the case of $V_{\mathrm{Ga}}^{q}$. However, they predict the stability of $V_{\mathrm{Sb}}^{+3}$ under high p-doping conditions, which exhibits a negative- $U$ transition to the +1 charge state. Again, we find a discrepancy when comparing to the work of Höglund et al. ${ }^{60}$ who studied InP, InAs, and InSb. They found that in InP, $V_{\text {In }}^{q}$ exists in the -3 and -4 charge states and in InAs only the -3 state, whereas for InSb, it undergoes a negative- $U$ transition from -1 to -3 states which are the only two stable states. These variations could stem in part from the different parameters used, such as the pseudopotentials and the supercell size. In particular, the charge corrections which are quite substantial for the highly charged states do not normally yield the same results when different schemes are used.

\section{CONCLUSIONS}

Vacancies in III-V semiconductors were investigated using first principle calculations. The formation energies were calculated for each vacancy, in different charge states, as a function of the Fermi level under stoichiometric conditions but also for III and V rich conditions. The correction scheme due to Freysoldt et al. ${ }^{23,24}$ was used throughout to correct for all charged defect interactions.

Considering vacancies at the intrinsic Fermi level, the formation energies decrease with increasing ion size and decreasing electronegativity of the group $\mathrm{V}$ ion. It is calculated that group III vacancies and group V vacancies have charge states in the range -3 to 0 and -3 to +1 , respectively, depending upon the Fermi level.

Fabrication of III-V semiconductors requires control of the concentrations of the defects that mediate transport, which includes vacancies. This can be achieved by altering the growth conditions, that is, making III or V rich or poor. The results presented here suggest, however, that for III-Sb, the growth conditions do not alter the preference for one vacancy over the other. For all other compounds, the vacancy that exhibits the dominant concentration can be changed at a specific value of the Fermi level but not across all Fermi level values.

The present systematic comparison of vacancy defects in the most important group III-V semiconductors aims to serve as a roadmap for future investigations.

\section{ACKNOWLEDGMENTS}

This research is based on a grant from King Abdullah University of Science and Technology (KAUST) which also provided the computational facilities (www.hpc.kaust.edu.sa) to carry out this work. H.T. wants to thank O. Chamseddine for useful discussions.

${ }^{1}$ R. Szweda, Gallium Arsenide, Electronics Materials and Devices. A Strategic Study of Markets, Technologies and Companies Worldwide 1999-2004 (Elsevier Science, 2000).

${ }^{2}$ J. S. Blakemore, J. Appl. Phys. 53, R123 (1982).

${ }^{3}$ P. S. Dutta, H. L. Bhat, and V. Kumar, J. Appl. Phys. 81, 5821 (1997).

${ }^{4}$ See www.itrs.net for the international technology roadmap for semiconductors, accessed April 2013.

${ }^{5}$ J. A. del Alamo, Nature 479, 317 (2011).

${ }^{6} \mathrm{~S}$. Adachi, Physical Properties of III-V Semiconductor Compounds (Wiley-Interscience Publication, 1992).

${ }^{7}$ D. N. Talwar and C. S. Ting, Phys. Rev. B 25, 2660 (1982).

${ }^{8}$ J. Hafner, J. Comput. Chem. 29, 2044 (2008).

${ }^{9}$ G. Kresse and J. Furthmüller, Phys. Rev. B 54, 11169 (1996).

${ }^{10}$ J. P. Perdew, K. Burke, and M. Ernzerhof, Phys. Rev. Lett. 77, 3865 (1996).

${ }^{11}$ P. E. Blöchl, Phys. Rev. B 50, 17953 (1994).

${ }^{12}$ H. J. Monkhorst and J. D. Pack, Phys. Rev. B 13, 5188 (1976).

${ }^{13}$ S. B. Zhang and J. E. Northrup, Phys. Rev. Lett. 67, 2339 (1991).

${ }^{14}$ F. El-Mellouhi and N. Mousseau, Phys. Rev. B 71, 125207 (2005).

${ }^{15}$ M. Freyss, T. Petit, and J.-P. Crocombette, J. Nucl. Mater. 347, 44 (2005).

${ }^{16}$ F. E. H. Hassan, A. Breidi, S. Ghemid, B. Amrani, H. Meradji, and O. Pagès, J. Alloys Compd. 499, 80 (2010).

${ }^{17}$ A. Hashibon and C. Elsässer, Phys. Rev. B 84, 144117 (2011).

${ }^{18}$ T. R. Paudel, S. S. Jaswal, and E. Y. Tsymbal, Phys. Rev. B 85, 104409 (2012).

${ }^{19}$ M. Youssef and B. Yildiz, Phys. Rev. B 86, 144109 (2012).

${ }^{20}$ R. Ramprasad, H. Zhu, P. Rinke, and M. Scheffler, Phys. Rev. Lett. 108, 066404 (2012).

${ }^{21}$ H.-P. Komsa, T. T. Rantala, and A. Pasquarello, Phys. Rev. B 86, 45112 (2012).

${ }^{22}$ G. Makov and M. C. Payne, Phys. Rev. B 51, 4014 (1995). 
${ }^{23}$ C. Freysoldt, J. Neugebauer, and C. G. Van de Walle, Phys. Rev. Lett. 102, 16402 (2009).

${ }^{24}$ C. Freysoldt, J. Neugebauer, and C. G. Van de Walle, Phys. Status Solidi B 248, 1067 (2011).

${ }^{25}$ N. D. M. Hine, K. Frensch, W. M. C. Foulkes, and M. W. Finnis, Phys. Rev. B 79, 24112 (2009).

${ }^{26}$ B. Delley, J. Phys. Chem. A 110, 13632 (2006).

${ }^{27}$ A. Jain, G. Hautier, S. P. Ong, C. J. Moore, C. C. Fischer, K. A. Persson, and G. Ceder, Phys. Rev. B 84, 045115 (2011).

${ }^{28} \mathrm{~W}$. M. Haynes, CRC Handbook of Chemistry and Physics, 92nd ed. (CRC Press, 2012).

${ }^{29}$ O. Madelung, Semiconductors: Data Handbook (Springer, Berlin, 2004).

${ }^{30}$ W. Chen, C. Tegenkamp, H. Pfnür, and T. Bredow, Phys. Rev. B 82, 104106 (2010).

${ }^{31}$ H.-P. Komsa and A. Pasquarello, Microelectron. Eng. 88, 1436 (2011).

${ }^{32}$ T. Shimazaki and Y. Asai, J. Chem. Phys. 132, 224105 (2010).

${ }^{33}$ Z. Y. Jiao, S. H. Ma, and Y. L. Guo, Comput. Theor. Chem. 970, 79 (2011).

${ }^{34}$ K. Seeger and E. Schonherr, Semicond. Sci. Technol. 6, 301 (1991).

${ }^{35}$ D. Ảberg, P. Erhart, A. J. Williamson, and V. Lordi, Phys. Rev. B 77, 165206 (2008).

${ }^{36}$ M.-H. Du, Phys. Rev. B 79, 45207 (2009).

${ }^{37}$ A. S. Jordan, A. R. Von Neida, R. Caruso, and C. K. Kim, J. Electrochem. Soc. 121, 153 (1974).

${ }^{38}$ A. Fazzio, L. M. Brescansin, and J. R. Leite, J. Phys. C 15, L1 (1982).

${ }^{39}$ L. Wang, J. A. Wolk, L. Hsu, E. E. Haller, J. W. Erickson, M. Cardona, T. Ruf, J. P. Silveira, and F. Briones, Appl. Phys. Lett. 70, 1831 (1997).

${ }^{40}$ A. Höglund, C. W. M. Castleton, and S. Mirbt, Phys. Rev. B 72, 195213 (2005).

${ }^{41}$ S. Y. Chiang and G. L. Pearson, J. Appl. Phys. 46, 2986 (1975).

${ }^{42}$ D. M. Taylor, D. O. Wilson, and D. H. Phillips, IEEE Proc., Part I: SolidState Electron Devices 127, 266 (1980).
${ }^{43}$ K. Hjort, J. Soderkvist, and J. A. Schweitz, J. Micromech. Microeng. 4, 1 (1994).

${ }^{44}$ J. T. Schick, C. G. Morgan, and P. Papoulias, Phys. Rev. B 66, 195302 (2002).

${ }^{45}$ P. A. Schultz and O. A. von Lilienfeld, Modell. Simul. Mater. Sci. Eng. 17, 84007 (2009).

${ }^{46}$ B.-H. Cheong and K. J. Chang, Phys. Rev. B 49, 17436 (1994).

${ }^{47}$ S. Pöykkö, M. J. Puska, and R. M. Nieminen, Phys. Rev. B 53, 3813 (1996).

${ }^{48}$ S. T. Murphy, A. Chroneos, R. W. Grimes, C. Jiang, and U. Schwingenschlögl, Phys. Rev. B 84, 184108 (2011).

${ }^{49}$ H. Bracht, S. P. Nicols, W. Walukiewicz, J. P. Silveira, F. Briones, and E. E. Haller, Nature 408, 69 (2000).

${ }^{50}$ A. Chroneos and H. Bracht, J. Appl. Phys. 104, 093714 (2008).

${ }^{51}$ A. Katz, Indium Phosphide and Related Materials: Processing, Technology, and Devices (Artech House Materials Library, Artech House, 1992).

${ }^{52}$ C. W. M. Castleton and S. Mirbt, Physica B 340, 407 (2003).

${ }^{53}$ C. W. M. Castleton and S. Mirbt, Phys. Rev. B 70, 195202 (2004).

${ }^{54} \mathrm{~W}$. LiMing, S. Fung, C. D. Beling, M. Fuchs, and A. P. Seitsonen, J. Phys.: Condens. Matter 10, 9263 (1998).

${ }^{55}$ D. G. Avery, D. W. Goodwin, and M. A. E. Rennie, J. Sci. Instrum. 34, 394 (1957).

${ }^{56}$ B. Cordero, V. Gomez, A. E. Platero-Prats, M. Reves, J. Echeverria, E. Cremades, F. Barragan, and S. Alvarez, Dalton Trans. 21, 2832 (2008).

${ }^{57}$ S. Sze and K. Ng, Physics of Semiconductor Devices (Wiley, 2006).

${ }^{58}$ J. E. Northrup and S. B. Zhang, Phys. Rev. B 50, 4962 (1994).

${ }^{59}$ V. Virkkala, V. Havu, F. Tuomisto, and M. J. Puska, Phys. Rev. B 86, 144101 (2012).

${ }^{60}$ A. Höglund, C. W. M. Castleton, M. Göthelid, B. Johansson, and S. Mirbt, Phys. Rev. B 74, 75332 (2006). 